\title{
The Validation of Questionnaire on End of Life Care Knowledge, Perceptions and Preferences among End Stage Renal Disease Patients on Haemodialysis in Kuantan
}

Nur Raziana Rozi ${ }^{1}$, Wan Ahmad Syahril Rozli Wan Ali ${ }^{1}$, Che Rosle Draman ${ }^{1}$, Hafizah Pasi $^{2}$

${ }^{1}$ Department of Internal Medicine, Kuliyyah of Medicine, International Islamic University Malaysia, Kuantan, Pahang, Malaysia

${ }^{2}$ Department of Community Medicine, Kuliyyah of Medicine, International Islamic University Malaysia, Kuantan, Pahang, Malaysia.

Introduction: There has been an increasing awareness on the end of life care for end stage renal disease (ESRD) patients and patients' factors have been identified as pivotal elements in directing its implementation. Questionnaire has been a useful tool by researchers to assess on patients' knowledge, perceptions and preferences on end of life care. However, up to date, there is no validated questionnaire developed in Malaysia for such purposes. Thus, this study aims to develop and validate questionnaires on end of life care knowledge, perceptions and preferences among ESRD patients in Kuantan. Materials and method: A cross-sectional study among haemodialysis (HD) patients aged 18 and above from 14 out-patient HD centres was performed. This study was divided into 4 phases i) developing the questionnaire involving related specialists and pre testing, ii) improving and editing the questionnaire and redistribution to 50 respondents for the pilot study, iii) factor analysis and iv) internal consistency reliability testing of the questionnaire. Results: The result of Factor analysis with Varimax rotation performed identified 3 domains for the 41 items, with 10 to 20 items in each domain. All the 41 analyzed items had a good factor loading of more than 0.4 with the lowest value of 0.421 and were nicely fit into 3 respective domains; knowledge, perception and preference. Internal consistency reliability analysis performed indicates that Cronbach's a was between 0.5 to 0.7 for all factors which were higher than the level set for this study which is 0.5. Conclusion: The questionnaire is successfully validated and considered as a useful tool to be used. Nonetheless, improvement will still be necessary from time to time to ensure its relevance especially if it is to be used by different populations or countries of different background than Malaysia. 